\title{
STOCHASTIC EPIDEMIC MODELS IN STRUCTURED POPULATIONS FEATURING DYNAMIC VACCINATION AND ISOLATION
}

\author{
FRANK BALL, ${ }^{* * *}$ \\ PHILIP D. O'NEILL **** AND \\ JAMES PIKE, ${ }^{* * * * *}$ University of Nottingham
}

\begin{abstract}
We consider a stochastic model for the spread of an SEIR (susceptible $\rightarrow$ exposed $\rightarrow$ infective $\rightarrow$ removed) epidemic among a population of individuals partitioned into households. The model incorporates both vaccination and isolation in response to the detection of cases. When the infectious period is exponential, we derive an explicit formula for a threshold parameter, and analytic results that enable computation of the probability of the epidemic taking off. These quantities are found to be independent of the exposure period distribution. An approximation for the expected final size of an epidemic that takes off is obtained, evaluated numerically, and found to be reasonably accurate in large populations. When the infectious period is not exponential, but has an increasing hazard rate, we obtain stochastic comparison results in the case where the exposure period is fixed. Our main result shows that as the exposure period increases, both the severity of the epidemic in a single household and the threshold parameter decrease, under certain assumptions concerning isolation. Corresponding results for infectious periods with decreasing hazard rates are also derived.
\end{abstract}

Keywords: Epidemic; stochastic epidemic; vaccination; bioterrorism; coupling; final outcome

2000 Mathematics Subject Classification: Primary 92D30

Secondary $60 \mathrm{~K} 99$

\section{Introduction}

This paper is concerned with a model for the spread of an infectious disease through a population structured into household units. Our motivation comes from considerations in bioterror modelling (see below) regarding real-time response strategies to infectious diseases. To this end, the model incorporates both vaccination and isolation of households, these events being triggered by the appearance of symptoms in individuals. In these respects our modelling is distinct from the following two cases:

(i) the modelling of new emerging diseases, in which vaccines are usually not available;

(ii) the modelling of endemic diseases in which mass or age-targeted vaccination takes place in a programmed way that is independent of the course of a particular outbreak.

Received 17 June 2005; revision received 15 June 2007.

* Postal address: School of Mathematical Sciences, University of Nottingham, University Park, Nottingham NG7 2RD, UK.

** Email address: frank.ball@nottingham.ac.uk

*** Email address: philip.o'neill@ nottingham.ac.uk

**** Email address: james.pike@ adelphigroup.com 
Our model has two levels of mixing (see Ball et al. (1997)) in the sense that individuals may have potentially infectious contacts with individuals within their own household, and also with individuals in the population at large, according to two rate parameters of different orders of magnitude. The model also incorporates an exposed (latent) period for the disease.

In recent years there has been considerable interest in the use of mathematical models for the evaluation of possible response strategies in the face of possible bioterrorism activity; see, e.g. Halloran et al. (2002), Kaplan et al. (2002), Eubank et al. (2004), and the references therein. It is reasonable to assume that any infectious agents employed in bioterror actions are likely to be well known in an epidemiological sense. In particular, a vaccine may exist, so that one pertinent control issue is to ascertain the most effective use of such a vaccine, possibly in conjunction with other control measures such as quarantine, movement restrictions, etc. Smallpox has been prominent among modelling studies; for example, Halloran et al. (2002) and Kaplan et al. (2002) both considered the merits of targeted versus mass vaccination, assuming various outbreak scenarios for this disease. The methods developed in this paper are also relevant to modelling targeted antiviral prophylaxis strategies for containing an emerging influenza pandemic, where households are a key mixing group; see, for example, the simulation models of Longini et al. (2004) and Ferguson et al. (2005).

It is typically the case that the bioterrorism and pandemic influenza models referred to above are highly complex in nature, as driven by concerns for realism. This in turn makes mathematical analysis virtually impossible, so that progress must be made by either numerical or simulation studies. In the present paper our aim is more towards mathematical analysis, and to this end we consider a simplified model which nevertheless captures many of the features of the more complex models that have been utilised to date.

The paper is organised as follows. The model is defined in Section 2 and its threshold behaviour is determined in terms of the severity of an associated single household epidemic. Section 3 contains results when an exponentially distributed infectious period is assumed. The threshold parameter and the probability of the epidemic taking off are obtained, and the final outcome of epidemics that do take off is investigated. The first two quantities do not depend on the exposure period distribution. Section 4 focuses on the comparison of results for epidemics with different exposure period distributions and a common infectious period distribution, which need not be exponential. Finally, some brief concluding comments are given in Section 5.

\section{Model and threshold behaviour}

\subsection{Model}

The model used is a modification of an SEIR (susceptible $\rightarrow$ exposed $\rightarrow$ infective $\rightarrow$ removed) epidemic model among a closed population. Thus, at any time, each individual is in one of four states: susceptible, exposed, infective, or removed. The population is made up of $N$ individuals partitioned into $m$ groups, or households, of size $n$. Initially a small number of individuals are infectives and the remaining individuals are susceptibles. A susceptible individual becomes an exposed individual if contact is made with an infective in the manner described below. An exposed individual remains so for a period of time distributed according to some arbitrary but specified random variable $T_{\mathrm{E}}$ and then becomes an infective. An infective individual is infectious for a period of time distributed according to some arbitrary but specified random variable $T_{\mathrm{I}}$ and is then removed. Removed individuals play no further role in the epidemic process. A given infective makes global contacts with any given susceptible in the population at the points of a homogeneous Poisson process having rate $\lambda_{\mathrm{G}} / N$ and, 
additionally, it makes local contacts with any given susceptible in its household at the points of a homogeneous Poisson process having rate $\lambda_{\mathrm{L}}$. Each such contact immediately results in the susceptible becoming exposed. All the Poisson processes describing infectious contacts, as well as the random variables describing exposed and infectious periods, are assumed to be independent. The epidemic continues until there are no more exposed or infective individuals present in the population.

Vaccination and isolation policies are incorporated into the model. When a removal occurs in a household, all other members of that particular household are vaccinated. A susceptible individual, when vaccinated, immediately makes the transition from susceptible to removed. Thus, the vaccine is assumed to be completely effective. Vaccination has no effect on individuals in the three other states. This corresponds to an assumption that vaccination has no effect on an individual who has already been contacted by an infective. Additionally, for $j=1,2, \ldots$, at the time of the $j$ th removal in a particular household there is, independently of all previous events, a probability $p_{j}$ of the household being isolated. Infective individuals in an isolated household are unable to make global contacts. An individual's infectious period is therefore made up of two parts, $T_{\mathrm{I}}^{+}$and $T_{\mathrm{I}}^{-}$, where $T_{\mathrm{I}}^{+}$is the length of time the infected individual remains in the infective state whilst its household is not isolated and $T_{\mathrm{I}}^{-}$is the length of time the infected individual remains in the infective state whilst its household is isolated. We refer to $T_{\mathrm{I}}^{+}$and $T_{\mathrm{I}}^{-}$as the active and inactive parts of an individual's infectious period, respectively.

\subsection{Threshold behaviour}

If the number of households is large and the number of initial infectives is small, then during the early stages of the epidemic the probability that a global contact is with an individual residing in a previously infected household is small. Hence, the initial behaviour of the epidemic may be approximated by a process in which each global contact is made with an individual in an otherwise completely susceptible household. The process of infected households in this approximating process follows a branching process.

Consider a completely susceptible household in this approximating process into which infection is introduced by a global contact. The infected individual will initiate a realisation of a single household epidemic which does not receive any further global contacts owing to the assumption that all such contacts are made with completely susceptible households. The number of global contacts that emanate from this single household epidemic, $R$ say, is the offspring random variable for the approximating branching process.

This approximation of the epidemic process may be made fully mathematically rigorous by considering a sequence of epidemics in which $m \rightarrow \infty$ whilst $n$ is held fixed, and using a coupling argument; cf. Ball (1996). A threshold theorem for the epidemic process can then be obtained by saying that a global epidemic occurs if in the limit as $m \rightarrow \infty$ the epidemic infects infinitely many households, i.e. if the branching process does not go extinct. Let $R_{*}=\mathrm{E}[R]$ and $f(s)=\mathrm{E}\left[s^{R}\right]$ be the mean and probability generating function of $R$, respectively. Then, by standard branching process theory, a global epidemic may occur only if $R_{*}>1$, so $R_{*}$ is a threshold parameter for the epidemic process. Moreover, if $R_{*}>1$ and the epidemic is started by $a$ initial infectives who reside in distinct households, then the probability of a global epidemic is $1-\tau^{a}$, where $\tau$ is the root of $f(s)=s$ in $(0,1)$. For other configurations of initial infectives, the probability of a global epidemic can be found by conditioning on the size of the first generation in the branching process (cf. Ball et al. (1997)); note that in this case the offspring law of initial individuals in the branching process who correspond to households with more than 1 initial infective is different from that of individuals in subsequent generations. 
Consider a single household epidemic in the approximating branching process. Let $T_{\mathrm{A}}$ be the sum of the active parts of the infectious periods of all individuals who are infected in that household. We refer to $T_{\mathrm{A}}$ as the active severity of the single household epidemic. Note that $T_{\mathrm{A}}$ is not the same as the corresponding sum of both active and inactive parts, i.e. the integral under the trajectory of infectives in the household. Following Picard and Lefèvre (1990), we refer to this latter quantity as the severity of the single household epidemic. Since infectives make global contacts at rate $\lambda_{\mathrm{G}}$ throughout the active parts of their infectious periods, the total number of global contacts that emanate from this household follows a Poisson distribution with random mean $\lambda_{\mathrm{G}} T_{\mathrm{A}}$. Hence, $R_{*}=\lambda_{\mathrm{G}} \mathrm{E}\left[T_{\mathrm{A}}\right]$ and $f(s)=\psi\left(\lambda_{\mathrm{G}}(1-s)\right)$, where $\psi(\theta)=\mathrm{E}\left[\mathrm{e}^{-\theta T_{\mathrm{A}}}\right]$ is the moment generating function of $T_{\mathrm{A}}$.

\section{Exponential infectious period}

In this section $T_{\mathrm{E}}$ has an arbitrary but specified distribution and $T_{\mathrm{I}} \sim \operatorname{Exp}(\gamma)$, i.e. $T_{\mathrm{I}}$ is exponentially distributed with mean $\gamma^{-1}$. Explicit expressions are derived for $\mathrm{E}\left[T_{\mathrm{A}}\right]$ and $\psi(\theta)$, enabling the threshold parameter $R_{*}$ and the probability of a global epidemic to be determined. The final size of a global epidemic is also investigated.

\subsection{Calculation of $\boldsymbol{R}_{*}$ and probability of a global epidemic}

For $t \geq 0$, let $\boldsymbol{X}(t)=(S(t), E(t), I(t), R(t))$, where $S(t), E(t), I(t)$, and $R(t)$ respectively denote the numbers of susceptible, exposed, infective, and removed individuals at time $t$ in the above single household epidemic. The time $t=0$ is the start of the epidemic and, since we assume that the population initially consists only of infectives and susceptibles, $E(0)=R(0)=0$. Consider the following random time scale transformation of $\{X(t)\}=$ $\{X(t): t \geq 0\}$; cf. Watson (1980). For $t \geq 0$, let $\chi(t)=\int_{0}^{t} I(u) \mathrm{d} u$ be the severity of the epidemic over $[0, t]$. For $u \in\left[0, T_{\mathrm{A}}\right]$, let $\mathrm{U}(u)=\min \{t \geq 0: \chi(t)=u\}$ and let $\tilde{\boldsymbol{X}}(u)=(\tilde{S}(u), \tilde{E}(u), \tilde{I}(u), \tilde{R}(u))=\boldsymbol{X}(\mathrm{U}(u))$. The process $\{\tilde{\boldsymbol{X}}(u)\}=\left\{\tilde{\boldsymbol{X}}(u): 0 \leq u \leq T_{\mathrm{A}}\right\}$ is a random time scale transformation of $\{X(t)\}$, obtained by running the clock at rate $I(t)^{-1}$ when $I(t)>0$ and stopping the clock when $I(t)=0$. Observe that in the transformed process $\{\tilde{\boldsymbol{X}}(u)\}$,

(i) removals occur at the points of a homogeneous Poisson process with rate $\gamma$,

(ii) the times of these removals give the severity up until the corresponding removals in $\{\boldsymbol{X}(t)\}$

(iii) independently of the removal process, susceptible individuals are infected independently at rate $\lambda_{\mathrm{L}}$, provided that $\tilde{I}(u)>0$.

Let $T_{0}$ be the time of the first removal in $\{\tilde{\boldsymbol{X}}(u)\}$ and let $D=\tilde{E}\left(T_{0}\right)+\tilde{I}\left(T_{0}\right)$ be the number of exposed and infective individuals remaining just after that first removal occurs. Then (i) above implies that $T_{0} \sim \operatorname{Exp}(\gamma)$ and (iii) implies that $D \mid T_{0} \sim \operatorname{Bin}\left(n-1,1-\mathrm{e}^{-\lambda_{\mathrm{L}} T_{0}}\right)$. No new infections occur after time $T_{0}$ in $\{\tilde{X}(u)\}$ due to the vaccination scheme. Let $D^{\prime}$ be the number of removals that occur after time $T_{0}$ in $\{\tilde{\boldsymbol{X}}(u)\}$ before the household is isolated, so $D^{\prime}=0$ if the household is isolated when the first removal occurs. It follows, from (i) and (ii) above, that the active severity $T_{\mathrm{A}}$ admits the representation

$$
T_{\mathrm{A}}=T_{0}+T_{\mathrm{R}}
$$


where

$$
T_{\mathrm{R}}=\sum_{i=1}^{D^{\prime}} W_{i}
$$

$W_{1}, W_{2}, \ldots, W_{n-1}$ are independent $\operatorname{Exp}(\gamma)$ random variables which are independent of $\left(T_{0}, D, D^{\prime}\right)$ and the sum is 0 if $D^{\prime}=0$. Note that $T_{\mathrm{R}}$ is the severity generated in the household after the first removal. Note also that the distribution of the active severity $T_{\mathrm{A}}$ is invariant to the distribution of the exposed period $T_{\mathrm{E}}$. Thus, the threshold parameter $R_{*}$ and the probability of a global epidemic do not depend on the distribution of $T_{\mathrm{E}}$.

For $k=0,1, \ldots, n-1$ and $i=0,1, \ldots, k$, let $p_{k i}=\mathrm{P}\left(D^{\prime}=i \mid D=k\right)$. Then

$$
p_{k i}= \begin{cases}p_{i+1} \prod_{j=1}^{i}\left(1-p_{j}\right) & \text { if } i<k, \\ \prod_{j=1}^{k}\left(1-p_{j}\right) & \text { if } i=k,\end{cases}
$$

where the products are 1 if vacuous. For $k=0,1, \ldots, n-1$, let $\mu_{k}=\mathrm{E}\left[D^{\prime} \mid D=\right.$ $k]=\sum_{i=0}^{k} i p_{k i}$ and $\psi_{k}(\theta)=\mathrm{E}\left[\mathrm{e}^{-\theta T_{\mathrm{R}}} \mid D=k\right]=\sum_{i=0}^{k} p_{k i} \phi(\theta)^{i}, \theta \geq 0$, where $\phi(\theta)=$ $\gamma /(\gamma+\theta)$. Recall that $T_{0} \sim \operatorname{Exp}(\gamma)$ and $D \mid T_{0} \sim \operatorname{Bin}\left(n-1,1-\mathrm{e}^{-\lambda_{\mathrm{L}} T_{0}}\right)$. Conditioning on $T_{0}$, it follows, from (1) and (2), that

$$
\begin{aligned}
\mathrm{E}\left[T_{\mathrm{A}}\right] & =\mathrm{E}\left[T_{0}\right]+\mathrm{E}\left[W_{1}\right] \mathrm{E}\left[\mathrm{E}\left[D^{\prime} \mid T_{0}\right]\right] \\
& =\frac{1}{\gamma}\left\{1+\mathrm{E}\left[\sum_{k=0}^{n-1}\left(\begin{array}{c}
n-1 \\
k
\end{array}\right)\left(1-\mathrm{e}^{-\lambda_{\mathrm{L}} T_{0}}\right)^{k} \mathrm{e}^{-(n-1-k) \lambda_{\mathrm{L}} T_{0}} \mu_{k}\right]\right\} \\
& =\frac{1}{\gamma}+\frac{1}{\gamma} \sum_{k=0}^{n-1}\left(\begin{array}{c}
n-1 \\
k
\end{array}\right) \mu_{k} \sum_{l=0}^{k}\left(\begin{array}{l}
k \\
l
\end{array}\right)(-1)^{l} \mathrm{E}\left[\mathrm{e}^{-(n-1-k+l) \lambda_{\mathrm{L}} T_{0}}\right] .
\end{aligned}
$$

Note that $\mathrm{E}\left[\mathrm{e}^{-\theta T_{0}}\right]=\phi(\theta), \theta \geq 0$, and recall that $R_{*}=\lambda_{\mathrm{G}} \mathrm{E}\left[T_{\mathrm{A}}\right]$. Substituting $i=n-1-k+l$ in the second sum in (3) and changing the order of summation yields, after a little algebra,

$$
R_{*}=\frac{\lambda_{\mathrm{G}}}{\gamma}\left[1+\sum_{i=0}^{n-1}\left(\begin{array}{c}
n-1 \\
i
\end{array}\right) \phi\left(i \lambda_{\mathrm{L}}\right) \sum_{j=0}^{i}\left(\begin{array}{l}
i \\
j
\end{array}\right)(-1)^{i-j} \mu_{n-1-j}\right] \text {. }
$$

Turning to $\psi(\theta)=\mathrm{E}\left[\mathrm{e}^{-\theta T_{\mathrm{A}}}\right]$, by conditioning on $T_{0}$ it follows, from (1), that

$$
\psi(\theta)=\mathrm{E}\left[\mathrm{e}^{-\theta T_{0}} \mathrm{E}\left[\mathrm{e}^{-\theta T_{\mathrm{R}}} \mid T_{0}\right]\right] .
$$

Conditioning on $D$ and using (2) yields

$$
\mathrm{E}\left[\mathrm{e}^{-\theta T_{\mathrm{R}}} \mid T_{0}\right]=\sum_{k=0}^{n-1}\left(\begin{array}{c}
n-1 \\
k
\end{array}\right)\left(1-\mathrm{e}^{-\lambda_{\mathrm{L}} T_{0}}\right)^{k} \mathrm{e}^{-(n-1-k) \lambda_{\mathrm{L}} T_{0}} \psi_{k}(\theta)
$$

whence,

$$
\begin{aligned}
\psi(\theta) & =\mathrm{E}\left[\mathrm{e}^{-\theta T_{0}} \sum_{k=0}^{n-1}\left(\begin{array}{c}
n-1 \\
k
\end{array}\right) \psi_{k}(\theta) \sum_{l=0}^{k}\left(\begin{array}{l}
k \\
l
\end{array}\right)(-1)^{l} \mathrm{e}^{-(n-1-k+l) \lambda_{\mathrm{L}} T_{0}}\right] \\
& =\sum_{k=0}^{n-1}\left(\begin{array}{c}
n-1 \\
k
\end{array}\right) \psi_{k}(\theta) \sum_{l=0}^{k}\left(\begin{array}{l}
k \\
l
\end{array}\right)(-1)^{l} \phi\left((n-1-k+l) \lambda_{\mathrm{L}}+\theta\right)
\end{aligned}
$$




$$
=\sum_{i=0}^{n-1}\left(\begin{array}{c}
n-1 \\
i
\end{array}\right) \phi\left(i \lambda_{\mathrm{L}}+\theta\right) \sum_{j=0}^{i}\left(\begin{array}{l}
i \\
j
\end{array}\right)(-1)^{i-j} \psi_{n-1-j}(\theta)
$$

where the final step follows using similar algebraic manipulations to those used in the derivation of (4). Recall that the offspring probability generating function for the approximating process is $f(s)=\psi\left(\lambda_{\mathrm{G}}(1-s)\right)$. Thus, (5) enables the probability of a global epidemic to be computed numerically.

3.1.1. Constant isolation probability. The formulae for $R_{*}$ and $\psi(\theta)$ simplify if the isolation probability is constant. Suppose that $p_{i}=p, i=1,2, \ldots, n$. Then, for $k=0,1, \ldots$, $\mathrm{P}\left(D^{\prime} \geq i \mid D=k\right)=(1-p)^{i}, i=0,1, \ldots, k$, and, using $\mu_{k}=\mathrm{E}\left[D^{\prime} \mid D=k\right]=$ $\sum_{i=1}^{k} \mathrm{P}\left(D^{\prime} \geq i \mid D=k\right)$, we obtain

$$
\mu_{k}= \begin{cases}k & \text { if } p=0, \\ \frac{(1-p)\left[1-(1-p)^{k}\right]}{p} & \text { if } 0<p \leq 1 .\end{cases}
$$

Substituting these formulae for $\mu_{k}$ into (4) yields, after a little algebra,

$$
R_{*}= \begin{cases}\frac{\lambda_{\mathrm{G}}}{\gamma}\left[1+\frac{(n-1) \lambda_{\mathrm{L}}}{\lambda_{\mathrm{L}}+\gamma}\right] & \text { if } p=0, \\
\frac{\lambda_{\mathrm{G}}}{p}\left[\frac{1}{\gamma}-\sum_{i=0}^{n-1}\left(\begin{array}{c}
n-1 \\
i
\end{array}\right) \frac{(1-p)^{n-i} p^{i}}{i \lambda_{\mathrm{L}}+\gamma}\right] & \text { if } 0<p \leq 1 .\end{cases}
$$

A similar simplification is possible for $\psi(\theta)$. Omitting the details, we obtain

$$
\psi(\theta)=\frac{\gamma}{\theta+\gamma p}\left[p+\frac{(1-p) \theta}{(\gamma+\theta)^{n-1}} \sum_{i=0}^{n-1}\left(\begin{array}{c}
n-1 \\
i
\end{array}\right) \frac{[\gamma(1-p)]^{n-i-1}(\theta+\gamma p)^{i}}{i \lambda_{\mathrm{L}}+\gamma+\theta}\right], \quad \theta \geq 0 .
$$

Note that if $p=1$, so a household is isolated as soon as a removal occurs in it, then $R_{*}=\lambda_{\mathrm{G}} / \gamma$ and $\psi(\theta)=\gamma /(\gamma+\theta)$. Thus, a global epidemic can occur only if $\lambda_{\mathrm{G}}>\gamma$ and in that case, if initially there are $a$ initial infectives, all in distinct households, then the probability of a global epidemic is $1-R_{*}^{-a}$.

A related case of practical interest is $p_{1}=0, p_{2}=1$, meaning that households are isolated in the event of a second removal. We find that $\mu_{0}=0, \psi_{0}(\theta)=1$, and, for $k \geq 1, \mu_{k}=1$ and $\psi_{k}(\theta)=\phi(\theta)$. It follows, from (4) and (5), that

$$
\begin{aligned}
R_{*} & =\frac{\lambda_{\mathrm{G}}}{\gamma}\left[1+\frac{\lambda_{\mathrm{L}}(n-1)}{\gamma+\lambda_{\mathrm{L}}(n-1)}\right], \\
\psi(\theta) & =\phi(\theta)^{2}+\phi\left(\theta+\lambda_{\mathrm{L}}(n-1)\right)(1-\phi(\theta)), \quad \theta \geq 0 .
\end{aligned}
$$

3.1.2. Remarks and extensions. The above formulae for the threshold parameter $R_{*}$ indicate that isolation plays a key role in containing epidemic spread. Specifically, from (6) we see that if there is no isolation, i.e. $p=0$, then $R_{*}$ grows linearly in the household size $n$. Conversely, if isolation may occur then both (6) and (7) show that $R_{*}=O(1)$ as $n \rightarrow \infty$ and, in particular, $R_{*}$ is bounded above independently of household size. Note also that if $p_{1}=1$, so that isolation always occurs, then $R_{*}$ is independent of household size. This last fact is essentially a consequence of assuming an exponential infectious period and does not hold in the nonexponential case. 
Finally, the threshold parameter can be easily derived in the case where the population consists of households of unequal sizes. Specifically, for $k=1,2, \ldots$, let $\alpha_{k}$ denote the proportion of households in the population containing $k$ susceptibles at the start of the epidemic, and let $\tilde{\alpha}_{k}=k \alpha_{k} / \sum_{i \geq 1} i \alpha_{i}$ denote the probability that a randomly selected individual resides in a household of size $k$. Then it can be shown (cf. Ball et al. (1997)) that the threshold parameter for this model is

$$
R_{*}=\sum_{k=1}^{\infty} \tilde{\alpha}_{k} R_{*}^{(k)},
$$

where, for $k=1,2, \ldots, R_{*}^{(k)}$ is the $R_{*}$ in (4) with $n=k$.

\subsection{Approximation to final size}

The final size, $Z$ say, of the epidemic is the number of susceptible individuals that are infected during its course. Using the methodology of Ethier and Kurtz (1986, Chapter 11), it is possible to obtain numerical estimates of $\mathrm{E}(Z)$ and $\operatorname{var}(Z)$, conditional upon a global epidemic. This is achieved via a metapopulation deterministic approximation to the stochastic model, similar to that used by Ball (1999) for a households SIS (susceptible $\rightarrow$ infective $\rightarrow$ susceptible) model. Unfortunately, it is not possible to obtain analytic expressions for these quantities, although an approximation to $\mathrm{E}[Z]$ can be obtained by considering a modification of the original epidemic process in which, once a household is infected by a global contact, no further global contacts are made with that household. Note that this assumption was made when deriving the threshold behaviour of the original model, so the modification does not alter the threshold parameter or the probability of a global epidemic. The final size, $Z_{\mathrm{A}}$ say, of the modified process is clearly no larger than $Z$, and moreover if $\lambda_{\mathrm{G}}$ is sufficiently small then we would expect $Z_{\mathrm{A}}$ to be a reasonable approximation to $Z$.

Consider the approximating process, and suppose that the number of households $m$ is large, the initial number of infectives is small, and that a global epidemic occurs. Let $\rho$ denote the expected proportion of households that are ultimately infected. Note that if a household becomes infected then the active severity generated within it is given by $T_{\mathrm{A}}$ as defined in Section 3.1. It follows that the approximating process is equivalent to a homogeneously mixing SIR (susceptible $\rightarrow$ infective $\rightarrow$ removed) epidemic in which individuals correspond to households, the individual-to-individual infection rate is $\lambda_{\mathrm{G}} / m$, and the infectious period of an individual is distributed according to $T_{\mathrm{A}}$. We then have the following standard result (see, e.g. Andersson and Britton (2000, Equation (4.2))):

$$
1-\rho=\mathrm{e}^{-\lambda_{\mathrm{G}} \mathrm{E}\left[T_{\mathrm{A}}\right] \rho} .
$$

If $R_{*} \leq 1$, then $\rho=0$ is the only root of (8) in [0,1], while if $R_{*}>1$ then there is a unique second root giving the proportion of households ultimately infected by a global epidemic. Let $v$ denote the expected number of infections (including the initially contacted individual) that occur within a typical household that has been infected globally in the approximating process. Since within-household spread in the approximating process is identical to that in the branching process used to obtain $R_{*}, v=1+\mathrm{E}[D]$. Recall that $D \mid T_{0} \sim \operatorname{Bin}\left(n-1,1-\mathrm{e}^{-\lambda_{\mathrm{L}} T_{0}}\right)$ and $T_{0} \sim \operatorname{Exp}(\gamma)$. Thus,

$$
\begin{aligned}
v & =1+\mathrm{E}\left[\mathrm{E}\left[D \mid T_{0}\right]\right] \\
& =1+\mathrm{E}\left[(n-1)\left(1-\mathrm{e}^{-\lambda_{\mathrm{L}} T_{0}}\right)\right] \\
& =1+\frac{(n-1) \lambda_{\mathrm{L}}}{\lambda_{\mathrm{L}}+\gamma} .
\end{aligned}
$$



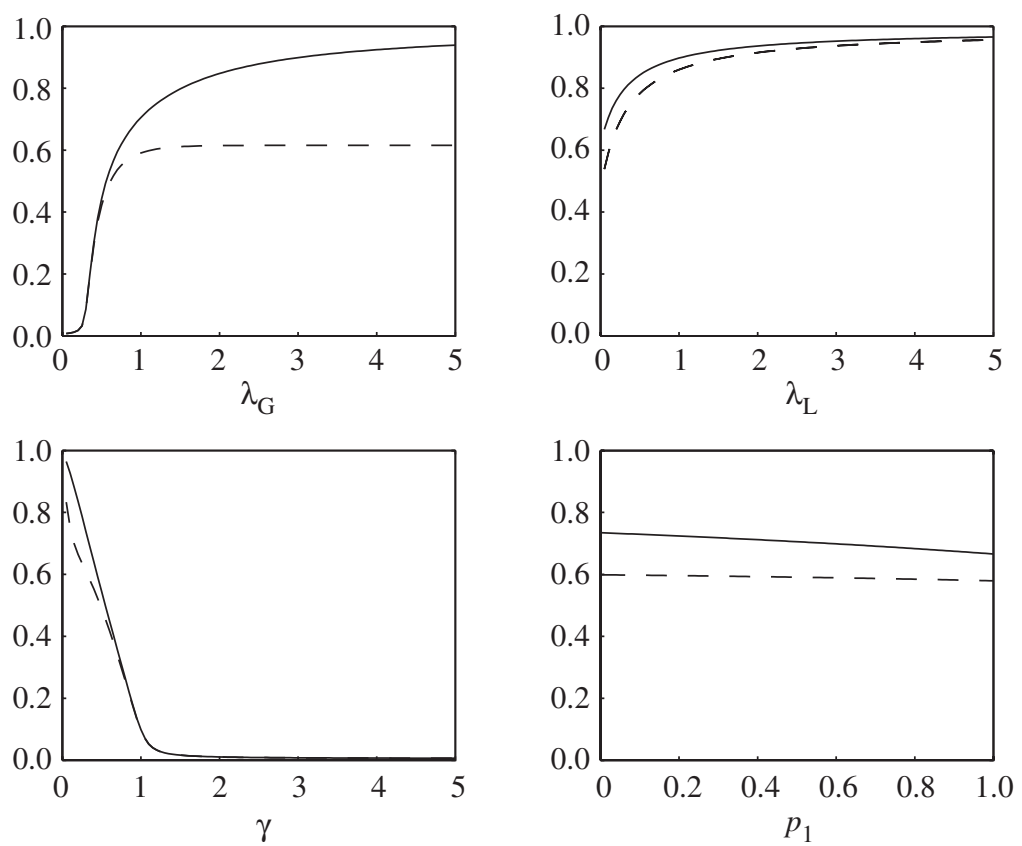

Figure 1: Asymptotic expected proportion of individuals infected by a global epidemic in the approximating and the original epidemic processes with $n=2, \lambda_{\mathrm{G}}=1, \lambda_{\mathrm{L}}=\frac{1}{10}, \mu=\frac{1}{8}, \gamma=\frac{1}{3}$, and $p_{1}=\frac{1}{2}$. The original epidemic process is denoted by solid lines and the approximating process is denoted by dashed lines.

It follows that the expected final size of the epidemic is approximately $\rho v m$. Note that such arguments can be extended to the case where the population consists of unequal household sizes, the key difference being that now the approximating process is multitype, where type corresponds to household size.

Figure 1 illustrates, for various values of $\lambda_{\mathrm{G}}, \lambda_{\mathrm{L}}, \gamma$, and $p_{1}$, the asymptotic $(m \rightarrow \infty)$ expected proportion of individuals ultimately infected by a global epidemic in the approximating and original epidemic processes, when $n=2, T_{\mathrm{E}} \sim \operatorname{Exp}(\mu)$, and $T_{\mathrm{I}} \sim \operatorname{Exp}(\gamma)$. Numerical solutions of the metapopulation deterministic approximation referred to above were used to obtain the plots for the original epidemic process. In each plot the effect of altering one parameter while the other parameters are held fixed is demonstrated. Altering $\mu$ has very little effect on $Z$ and no effect on $Z_{\mathrm{A}}$ so an example is not shown.

The difference in the expected proportion of individuals infected by a global epidemic in the approximating process and in the original process decreases as $\lambda_{L}$ becomes large. This is because once a household is infected by a global contact then, with high probability, all individuals of that household will become infected through local contacts if $\lambda_{\mathrm{L}}$ is sufficiently large. This reduces the effect of the assumption in the approximating process that a household can be globally contacted only once. Similarly, in either process, if $\gamma$ is close to 0 then, with high probability, all individuals in an infected household will become infected and if $\gamma$ is sufficiently large then, with high probability, no further infections will take place in an infected household. Increasing the value of $p_{1}$ reduces the difference in the expectations of the two processes 
because, as the probability of isolation increases, households in the original epidemic process will, in general, be isolated sooner and receive no further global contacts. Again, the behaviour of the original epidemic process becomes more like that of the approximating process. The difference in the expectations is most striking as $\lambda_{\mathrm{G}}$ becomes large. This, of course, is owing to the assumption in the approximating process that a household once infected globally will have no further global contacts made with it. As $\lambda_{\mathrm{G}}$ increases this assumption becomes less feasible and consequently $Z_{\mathrm{A}}$ is a less reliable approximation to $Z$. However, for sufficiently small $\lambda_{\mathrm{G}}$, $Z_{\mathrm{A}}$ appears to be a reasonable approximation to $Z$. Finally, changing the size of households does not alter the above qualitative conclusions, although the accuracy of the approximation decreases as the household size increases. Both of these observations were verified by further numerical investigations not presented here.

\section{Arbitrary infectious period}

In this section, a coupling argument is used to explore stochastic comparisons for the active severity and final outcome of single household epidemics with suitably ordered exposure period distributions, when the hazard function of $T_{\mathrm{I}}$ is monotone. This in turn leads to results concerning the dependency of the threshold parameter on the exposure period. Broadly speaking, we show that if $T_{\mathrm{I}}$ has an increasing hazard rate, then longer exposure periods lead to less overall severity, at least until the time of the first removal. The opposite conclusion holds if $T_{\mathrm{I}}$ has a decreasing hazard rate.

The intuition underlying such results is as follows. Consider a single household epidemic with one initial infective, suppose that $T_{\mathrm{I}}$ has an increasing hazard rate and that $T_{\mathrm{E}}=c$ is fixed. As $c$ increases, individuals spend longer in the exposed state, and at any given time the severity generated by that time decreases. However, at any fixed time the removal hazard rate due to the first infective remains the same, and moreover this hazard increases through time. This suggests that epidemics with longer exposure periods will generate less severity by the time of the first removal than those with shorter exposure periods.

The proofs of the results that we derive are sufficiently involved that we concentrate on conveying the key ideas in what follows. Full details are available in Ball et al. (2005). To start with, consider a single household epidemic initiated by a single infective, in which both $T_{\mathrm{E}}$ and $T_{\mathrm{I}}$ have arbitrary but specified distributions. We now give an alternative construction for this epidemic, motivated by Sellke (1983).

\subsection{Construction of the epidemic}

Initially, at time $t=0$, there exist a single infective individual and $n-1$ susceptible individuals who are labelled 0 and $1,2, \ldots, n-1$, respectively. For $j=1,2, \ldots, n-1$, let $Q_{j} \sim \operatorname{Exp}(1)$ denote the infection tolerance of susceptible $j$, and without loss of generality relabel the susceptibles so that $Q_{1} \leq Q_{2} \leq \cdots \leq Q_{n-1}$. For $t \geq 0$, let $I(t)$ be the number of infectives present at time $t$ and let $\bar{A}(t)=\int_{0}^{t} \lambda_{\mathrm{L}} I(u) \mathrm{d} u$ be the total infection pressure exerted on each susceptible up to time $t$. Susceptible $j$ becomes exposed when the total infection pressure reaches $Q_{j}$, i.e. at time $\inf \left\{s \geq 0: A(s) \geq Q_{j}\right\}$, and then remains so for a time $T_{\mathrm{E}, j} \sim T_{\mathrm{E}}$, after which they become infective. Note that the individuals are labelled according to the order in which they enter the exposed state.

For $j=0,1, \ldots, n-1$ and $t \geq 0$, let $h_{j}(t)$ denote the hazard rate at time $t$ of the infectious period of individual $j$, with the convention that $h_{j}(t)=0$ if individual $j$ is not infective at time $t$. Since the infectious periods of distinct individuals are mutually independent, the total hazard rate of a removal occurring at time $t$ is $h(t)=\sum_{j=0}^{n-1} h_{j}(t)$. Thus, the total removal 
pressure exerted on the household by time $t$ is

$$
V(t)=\int_{0}^{t} h(u) \mathrm{d} u .
$$

Let $\Pi:=\{\Pi(t): t \geq 0\}$ denote a homogeneous Poisson process with unit rate. Then removals occur at the points of the process $\{\Pi(V(t)): t \geq 0\}$. Let $W_{1}, W_{2}, \ldots, W_{n}$ be independent $\mathrm{U}(0,1)$ random variables. If a removal occurs at time $t$ then the probability that individual $i$ is removed is $h_{i}(t) / h(t), i=0,1, \ldots, n-1$. We therefore stipulate that if the $j$ th removal occurs at time $t$, then the individual removed has label $\min \left\{l \geq 0: \sum_{k=0}^{l} h_{k}(t) \geq W_{j} h(t)\right\}$.

As previously, at the time of the first removal all remaining individuals in the household are vaccinated, with the vaccine having the same action as described in Section 2.1. Additionally, for $j=1,2, \ldots, n$, the household is isolated at the time of the $j$ th removal if $U_{j} \leq p_{j}$, where $U_{j} \sim \mathrm{U}(0,1)$. The epidemic ceases when there is no exposed or infective individual present in the household. The $Q_{j} \mathrm{~s}, T_{\mathrm{E}, j} \mathrm{~s}, W_{j} \mathrm{~s}, U_{j} \mathrm{~s}$, and the Poisson process $\Pi$ are assumed to be mutually independent.

\subsection{Comparing epidemics with different exposed periods}

4.2.1. Preliminaries and technical results. We now restrict our attention to epidemics in which $T_{\mathrm{E}, j}=c \geq 0$ for all $j$, i.e. the exposed period is constant for all individuals. Consider two such epidemics, $E^{(1)}$ and $E^{(2)}$, with respective exposed periods $c$ and $c+\Delta$, where $|\Delta|$ is small, as clarified later. Let $\lambda(t)$ denote the hazard function of $T_{\mathrm{I}}$ and recall that $T_{\mathrm{I}}$ has an increasing failure rate if $\lambda(t)$ is a nondecreasing function of $t$. A decreasing failure rate is defined analagously. We shall derive a comparison result for the severity, at the time of the first removal, in the two epidemics under the assumption that $T_{\mathrm{I}}$ has an increasing failure rate. This is achieved via a coupling argument using the above construction.

Let $(\Omega, \mathcal{F}, \mathrm{P})$ be a probability space upon which is defined a unit rate Poisson process $\Pi$ and random vectors $\left(Q_{1}, Q_{2}, \ldots, Q_{n-1}\right),\left(W_{1}, W_{2}, \ldots, W_{n}\right)$, and $\left(U_{1}, U_{2}, \ldots, U_{n}\right)$, distributed as in Section 4.1. For $j=0, \ldots, n-1$, set $T_{\mathrm{E}, j}^{(1)}=c$ and $T_{\mathrm{E}, j}^{(2)}=c+\Delta$, where $\Delta>-c$.

Now construct epidemics $E^{(1)}$ and $E^{(2)}$ on $\Omega$ as follows. For $i=1,2, E^{(i)}$ is constructed using the $Q_{j} \mathrm{~s}, W_{j} \mathrm{~s}, T_{\mathrm{E}, j}^{(i)} \mathrm{s}, U_{j} \mathrm{~s}$, and $\Pi$. Thus, for a given $\omega \in \Omega$ both epidemics have the same tolerances to infection and equivalent removal processes, but individuals in $E^{(2)}$ remain in the exposed state for longer if $\Delta>0$ (shorter if $\Delta<0$ ). In the remainder of this section the explicit dependence on $\omega$ is often suppressed.

In this construction, if $\Delta>0$ and no removal has occurred in either epidemic, then $I^{(1)}(t) \geq I^{(2)}(t)$ and $\chi^{(1)}(t) \geq \chi^{(2)}(t)$, where $I^{(j)}(t)$ and $\chi^{(j)}(t)=\int_{0}^{t} I^{(j)}(u) \mathrm{d} u$, are respectively the number of infectives present at time $t$ and the severity generated up to time $t$ in epidemic $E^{(j)}$. Let $t_{\mathrm{R}}$ denote the time of the first removal in $E^{(1)}$.

Consider the epidemic $E^{(2)}$ without the removal of infected individuals and call this epidemic $E^{(*)}$. Define $t^{*}=t^{*}\left(t_{\mathrm{R}}\right)=\inf \left\{s \geq 0: \chi^{(*)}(s)=\chi^{(1)}\left(t_{\mathrm{R}}\right)\right\}$ to be the time at which the severity in $E^{(*)}$ reaches that of $E^{(1)}$ at the time of the first removal. We start with a technical lemma that is needed in the sequel. Its proof is straightforward via induction.

Lemma 1. Let $M>0$ be a positive integer, and suppose that $a(0), a(1), \ldots, a(M)$ is any nondecreasing sequence of nonnegative integers such that $a(0)=0$ and $a(j) \leq j$ for $j=$ $1,2, \ldots, M$. Define a sequence $\eta_{0}, \eta_{1}, \ldots, \eta_{M}$ by $\eta_{0}=0$ and

$$
\eta_{j}=\eta_{j-1}+(j+1)^{-1}\left(\tilde{\eta}_{j-1}-\eta_{j-1}+\Delta\right), \quad j=1,2, \ldots, M
$$


where $\tilde{\eta}_{j}=\eta_{a(j)}$ and $\Delta \geq 0$. Then, for $j=1,2, \ldots, M$,

$$
\eta_{j} \geq \eta_{j-1}, \quad \tilde{\eta}_{j}-\eta_{j}+\Delta \geq 0 .
$$

Recall that epidemic $E^{(1)}$ has exposure period $c$. The next result essentially shows that we can find a $\Delta>0$ such that epidemic $E^{(*)}$, with exposure period $c+\Delta$, has removal pressure at time $t^{*}$ that is no less than that of $E^{(1)}$ at time $t_{\mathrm{R}}$. Thus, the comparison occurs at times corresponding to equal severity. Furthermore, we can find a $\Delta<0$ with an appropriate converse conclusion holding. We provide a sketch proof of part (a); part (b) is similar and hence omitted.

Lemma 2. Suppose that $T_{\mathrm{I}}$ has an increasing failure rate.

(a) For $\mathrm{P}$-almost all $\omega \in \Omega$, for each $c \geq 0$ there exists $\Delta(\omega, c)>0$ such that $V^{(*)}\left(t^{*}, \omega\right) \geq$ $V^{(1)}\left(t_{\mathrm{R}}, \omega\right)$.

(b) For $\mathrm{P}$-almost all $\omega \in \Omega$, for each $c>0$ there exists $\Delta(\omega, c)<0$ such that $V^{(*)}\left(t^{*}, \omega\right) \leq$ $V^{(1)}\left(t_{\mathrm{R}}, \omega\right)$.

If $T_{\mathrm{I}}$ has a decreasing failure rate then the above conclusions hold with the inequalities involving $V^{(*)}$ and $V^{(1)}$ reversed.

Proof of Lemma 2(a). Note that $t^{*} \geq t_{\mathrm{R}}$ by construction. The result is straightforward if $t_{\mathrm{R}}=0$ or $t^{*}=t_{\mathrm{R}}$, so suppose, henceforth, that $t^{*}>t_{\mathrm{R}}$. For $j=1,2, \ldots, n-1$, let $s_{j}$ denote the time at which the $j$ th infectious individual (excluding the initial infective) appears in $E^{(1)}$. If $t_{j}$ is the time at which this individual was infected then $s_{j}=t_{j}+c$. Denote by $s_{j}^{*}$ and $t_{j}^{*}$ the corresponding times in $E^{(*)}$, so that $s_{j}^{*}=t_{j}^{*}+c+\Delta$. Let

$$
A=\left\{\omega \in \Omega: Q_{i} \neq Q_{j} \text { for } i \neq j, i, j=1,2, \ldots, n-1\right\} .
$$

Note that $\mathrm{P}(A)=1$. We assume henceforth that $\omega \in A$; whence, $s_{1}<s_{2}<\cdots<s_{m}<t_{\mathrm{R}}$, where $m=I^{(1)}\left(t_{\mathrm{R}}-\right)-1 \geq 1$, the latter inequality holding since $t^{*}>t_{\mathrm{R}}$.

The remainder of the proof relies intrinsically upon the relative behaviour of the two severity functions $\chi^{(1)}$ and $\chi^{(*)}$, as illustrated in Figure 2. During [0, $\left.t_{\mathrm{R}}\right), \chi^{(1)}$ is piecewise linear, with change-points at $s_{1}, s_{2} \ldots, s_{m}$ and gradients $\left(\chi^{(1)}(t)\right)^{\prime}=j$ for $t \in\left(s_{j-1}, s_{j}\right)$, where $s_{0}=0$. Thus, $\chi^{(1)}\left(s_{1}\right)<\chi^{(1)}\left(s_{2}\right)<\cdots<\chi^{(1)}\left(s_{m}\right)<\chi^{(1)}\left(t_{\mathrm{R}}\right)$. Similar comments apply to $\chi^{(*)}$. Note that, for clarity, the gradients in Figure 2 are not represented exactly.

In the sequel we shall be concerned with the quantity $t^{*}-t_{\mathrm{R}}$. To this end, define a sequence $\left(\delta_{j}\right)=\left(\delta_{1}, \delta_{2}, \ldots, \delta_{m}\right)$ as illustrated in Figure 2 , the point being that $t^{*}-t_{\mathrm{R}}=\delta_{m}$. Specifically, $\delta_{j}$ is the horizontal distance between $\chi^{(1)}$ and $\chi^{(*)}$ when both functions have common derivative $j+1$. This sequence is well defined provided that $\Delta$ is small enough, which we assume to be the case. We also require that $I^{(*)}\left(t^{*}-\right)=I^{(1)}\left(t_{\mathrm{R}}-\right)=m+1$, which again holds for small enough $\Delta$. Next, it can be shown, by induction, that the definition of $\eta_{j}$ in Lemma 1 corresponds to that of $\delta_{j}$, assuming that $a(j)=\left(\chi^{(1)}\left(t_{j}\right)\right)^{\prime}$, and $\tilde{\delta}_{j}=\delta_{a(j)}, j=1,2, \ldots, m$. The $a(j)$ s indicate the difference in infection times, in that $t_{j+1}^{*}-t_{j+1}=\delta_{a(j)}=\tilde{\delta}_{j}$ under the assumption that $\Delta$ is sufficiently small.

The initial infective is present in both $E^{(1)}$ just before time $t_{\mathrm{R}}$, and in $E^{(*)}$ just before $t^{*}$, since no removals occur in $E^{(*)}$. The severity in $E^{(1)}$ at time $t_{\mathrm{R}}$ is a sum of the contribution from the initial infective and the other $m$ infectives present at $t_{\mathrm{R}}-$. This and corresponding reasoning for $E^{(*)}$ gives

$$
\chi^{(1)}\left(t_{\mathrm{R}}\right)=t_{\mathrm{R}}+\sum_{j=1}^{m}\left(t_{\mathrm{R}}-s_{j}\right) \quad \text { and } \quad \chi^{(*)}\left(t^{*}\right)=t^{*}+\sum_{j=1}^{m}\left(t^{*}-s_{j}^{*}\right),
$$




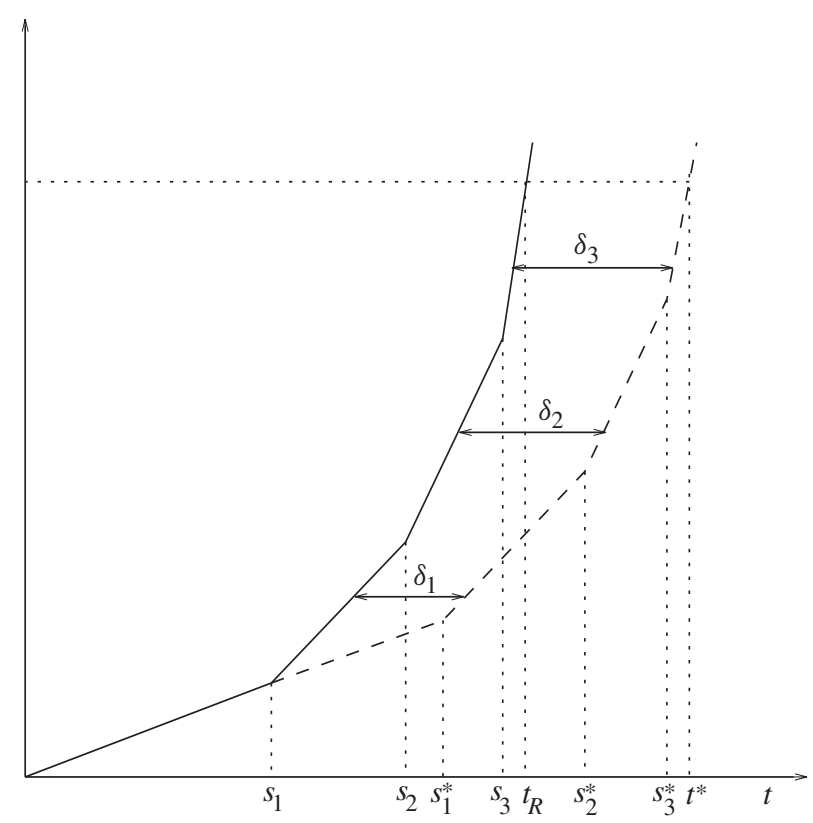

Figure 2: Typical realisation of the two severity functions $\chi^{(1)}$ (solid line) and $\chi^{(*)}$ (dashed line). Here $\delta_{m}=\delta_{3}=t^{*}-t_{\mathrm{R}}$. The horizontal dotted line indicates $\chi^{(1)}\left(t_{\mathrm{R}}\right)=\chi^{(*)}\left(t^{*}\right)$.

which, combined with the fact that $\chi^{(1)}\left(t_{\mathrm{R}}\right)=\chi^{(*)}\left(t^{*}\right)$, yields

$$
t^{*}-t_{\mathrm{R}}=\sum_{j=1}^{m}\left\{\left(t_{\mathrm{R}}-s_{j}\right)-\left(t^{*}-s_{j}^{*}\right)\right\}
$$

Similarly, the removal pressure in both epidemics can be written as a sum of the contributions from the initial infective and any other infectives. It follows that

$$
V^{(*)}\left(t^{*}\right)-V^{(1)}\left(t_{\mathrm{R}}\right)=\int_{t_{\mathrm{R}}}^{t^{*}} \lambda(u) \mathrm{d} u-\sum_{j=1}^{m} \int_{t^{*}-s_{j}^{*}}^{t_{\mathrm{R}}-s_{j}} \lambda(u) \mathrm{d} u .
$$

Recall now that $t^{*}-t_{\mathrm{R}}=\delta_{m}$, and that $\left(t_{\mathrm{R}}-s_{j}\right)-\left(t^{*}-s_{j}^{*}\right)=\Delta+\tilde{\delta}_{j-1}-\delta_{m}$. From Lemma 1 , the sequence $\left(\tilde{\delta}_{j}\right)$ is nondecreasing; hence, so is the sequence $\left(\left(t_{\mathrm{R}}-s_{j}\right)-\left(t^{*}-s_{j}^{*}\right)\right)$. Now, $t^{*}-t_{\mathrm{R}}>0$, so it follows, from (9), that there exists a $1 \leq k \leq m$ such that

$$
\begin{array}{ll}
\left(t_{\mathrm{R}}-s_{j}\right)-\left(t^{*}-s_{j}^{*}\right)<0 & \text { for } j<k, \\
\left(t_{\mathrm{R}}-s_{j}\right)-\left(t^{*}-s_{j}^{*}\right) \geq 0 & \text { for } m \geq j \geq k .
\end{array}
$$

Define

$$
V^{+}=\int_{t_{\mathrm{R}}}^{t^{*}} \lambda(u) \mathrm{d} u+\sum_{j=1}^{k-1} \int_{t_{\mathrm{R}}-s_{j}}^{t^{*}-s_{j}^{*}} \lambda(u) \mathrm{d} u \quad \text { and } \quad V^{-}=\sum_{j=k}^{m} \int_{t^{*}-s_{j}^{*}}^{t_{\mathrm{R}}-s_{j}} \lambda(u) \mathrm{d} u,
$$


where a sum is 0 if vacuous. From (10) it follows that $V^{(*)}\left(t^{*}\right)-V^{(1)}\left(t_{\mathrm{R}}\right)=V^{+}-V^{-}$. Now (9) implies that

$$
t^{*}-t_{\mathrm{R}}+\sum_{j=1}^{k-1}\left\{\left(t^{*}-s_{j}^{*}\right)-\left(t_{\mathrm{R}}-s_{j}\right)\right\}=\sum_{j=k}^{m}\left\{\left(t^{*}-s_{j}^{*}\right)-\left(t_{\mathrm{R}}-s_{j}\right)\right\}
$$

whence, the total lengths of the intervals of the integrals in $V^{+}$is equal to that in $V^{-}$. However, since $s_{j}<s_{j+1}$ for $j=1,2, \ldots, m-1, u \geq t_{\mathrm{R}}-s_{k-1}$ in all of the integrals in $V^{+}$, whilst $u \leq t_{\mathrm{R}}-s_{k}$ in all of the integrals in $V^{-}$. Therefore, $V^{+} \geq V^{-}$if $T_{\mathrm{I}}$ has an increasing hazard rate, and $V^{+} \leq V^{-}$if $T_{\mathrm{I}}$ has a decreasing hazard rate, as required.

4.2.2. Main results and discussion. We now turn our attention to the severity generated up until the time of the first removal, i.e. $T_{0}$, and view this quantity as a function of the latent period length, $c$, writing $T_{0}(c)$.

Theorem 1. Suppose that $T_{\mathrm{I}}$ has an increasing failure rate. Then, for $\mathrm{P}$-almost all $\omega \in \Omega$, $T_{0}(c)$ is nonincreasing on $[0, \infty)$. The reverse conclusion holds if $T_{\mathrm{I}}$ has a decreasing failure rate.

Proof. Suppose that $T_{\mathrm{I}}$ has an increasing failure rate. (The proof when $T_{\mathrm{I}}$ has a decreasing failure rate is similar, and hence omitted.) Fix $\omega \in A$ and let $c \geq 0$. Then by Lemma 2(a) there exists $\Delta=\Delta(\omega, c)>0$ such that $V^{(*)}\left(t^{*}\right) \geq V^{(1)}\left(t_{\mathrm{R}}\right)$. Let $t_{\mathrm{R}}^{(2)}$ denote the time of the first removal in $E^{(2)}$. Then $t_{\mathrm{R}}^{(2)} \leq t^{*}$, since $V^{(*)}$ and $V^{(2)}$ are nondecreasing and coincide over $\left[0, t_{\mathrm{R}}^{(2)}\right]$, and the same realisation of $\Pi$ is used to construct both $E^{(1)}$ and $E^{(2)}$. Hence, since $\chi^{(1)}, \chi^{(2)}$, and $\chi^{(*)}$ are nondecreasing, and $\chi^{(2)}$ and $\chi^{(*)}$ coincide over $\left[0, t_{\mathrm{R}}^{(2)}\right]$,

$$
T_{0}(c+\Delta)=\chi^{(2)}\left(t_{\mathrm{R}}^{(2)}\right)=\chi^{(*)}\left(t_{\mathrm{R}}^{(2)}\right) \leq \chi^{(*)}\left(t^{*}\right)=\chi^{(1)}\left(t_{\mathrm{R}}\right)=T_{0}(c) .
$$

Moreover, it is easily seen that Lemma 2(a) remains valid with $\Delta$ replaced by any smaller positive value, i.e. by any $0<x \leq \Delta$. We may thus conclude that, for any $c \geq 0$, there exists $\Delta_{1}=\Delta_{1}(c)>0$ such that $T_{0}(c) \geq T_{0}(c+x)$ for all $0 \leq x \leq \Delta_{1}$. A similar argument using Lemma 2(b) shows that, for any $c>0$, there exists $\Delta_{2}=\Delta_{2}(c)>0$ such that $T_{0}(c) \leq T_{0}(c-x)$ for all $0 \leq x \leq \Delta_{2}$. Finally, it can be shown by contradiction, using a bisection argument, that these two facts imply that $T_{0}(c)$ is nonincreasing on $[0, \infty)$, and the result follows.

Recall that, in the single household epidemic, $D$ is the number of initially susceptible individuals that are ultimately infected and $T_{\mathrm{A}}$ is the active severity. We view these quantities as functions of $c$ and write $D(c)$ and $T_{\mathrm{A}}(c)$, respectively.

Corollary 1. Suppose that $T_{\mathrm{I}}$ has an increasing failure rate. Then

(i) $D(c)$ is nonincreasing on $[0, \infty)$ for $\mathrm{P}$-almost all $\omega \in \Omega$,

(ii) if $p_{1}=1$ then $T_{\mathrm{A}}(c)$ is nonincreasing on $[0, \infty)$ for $\mathrm{P}$-almost all $\omega \in \Omega$,

(iii) if $p_{1}=1$ or $p_{i}=0, i=1,2, \ldots, n$, then $\mathrm{E}\left[T_{\mathrm{A}}(c)\right]$ is nonincreasing on $[0, \infty)$.

The reverse conclusions hold if $T_{\mathrm{I}}$ has a decreasing failure rate.

Proof. Part (i) follows from Theorem 1 on noting that $D(c)=\left|\left\{j: Q_{j} \leq \lambda_{\mathrm{L}} T_{0}(c)\right\}\right|$, where $|\cdot|$ denotes cardinality, since the same $Q_{j}$ s are used to construct the epidemic for different $c$. Part (ii) follows from Theorem 1 since $T_{\mathrm{A}}(c)=T_{0}(c)$ if $p_{1}=1$. 
For part (iii), if $p_{1}=1$ then the result is an immediate consequence of part (ii). Otherwise, label the individuals in the household $0,1, \ldots, n-1$, with 0 being the initial infective, and construct the epidemic as in Section 2.1 but without global infection. For $j=0,1, \ldots, n-1$, let $\alpha_{j}=1$ if individual $j$ is infected by the epidemic and let $\alpha_{j}=0$ otherwise, and also let $T_{\mathrm{I}, j}$ denote the infectious period of individual $j$ if they become infected. Note that if $p_{i}=0, i=$ $1,2, \ldots, n$, then $T_{\mathrm{I}, j}$ and $\alpha_{j}$ are independent $(j=0,1, \ldots, n-1)$ and $T_{\mathrm{A}}=\sum_{j=0}^{n-1} T_{\mathrm{I}, j} \alpha_{j}$. Thus,

$$
\mathrm{E}\left[T_{\mathrm{A}}\right]=(1+\mathrm{E}[D]) \mathrm{E}\left[T_{\mathrm{I}}\right],
$$

and the result follows using part (i).

Theorem 1 and Corollary 1 can be strengthened when $T_{\mathrm{I}}$ does not have an exponential distribution. In particular, it can be shown that if households are necessarily isolated when their first removal occurs, i.e. $p_{1}=1$, or there is no isolation, i.e. $p_{i}=0, i=1,2, \ldots, n$, then $\mathrm{E}\left[T_{\mathrm{A}}(c)\right]$ is strictly decreasing in $c$ if $T_{\mathrm{I}}$ has an increasing failure rate.

Recall that the threshold parameter $R_{*}$ of the households model described in Section 2.1 is given by $R_{*}=\lambda_{\mathrm{G}} \mathrm{E}\left[T_{\mathrm{A}}\right]$. Suppose that $T_{\mathrm{I}}$ has an increasing failure rate, and that either $p_{1}=1$ or $p_{i}=0, i=1,2, \ldots, n$. Then Corollary 1(iii) implies that, in obvious notation, $R_{*}(c)$ is nonincreasing on $[0, \infty)$. Thus, in particular, upper and lower bounds for $R_{*}(c)$ can be obtained by letting $c=0$ and $c \rightarrow \infty$, respectively.

Bounds for $R_{*}$ may be also derived for nonconstant exposure periods as follows. Suppose that $T_{\mathrm{E}}$ has an arbitrary but specified distribution and construct epidemics $E^{(1)}$ and $E^{(2)}$ as in Section 4.2.1, where, for $j=1,2, \ldots, n-1, T_{\mathrm{E}, j}^{(1)}$ is distributed according to $T_{\mathrm{E}}$ and $T_{\mathrm{E}, j}^{(2)}=\infty$. Note that $E^{(2)}$ describes a situation in which only the initial infective contributes to the severity and the removal pressure. By similar arguments to those in the proof of Lemma 2, it can be shown that for P-almost all $\omega \in \Omega, V^{(*)}\left(t^{*}\right) \geq V^{(1)}\left(t_{\mathrm{R}}\right)$ if $T_{\mathrm{I}}$ has an increasing failure rate. Thus, if $p_{1}=1$ or $p_{i}=0, i=1,2, \ldots, n$, letting $T_{\mathrm{E}} \rightarrow \infty$ yields a lower bound for $R_{*}$. Similarly, an upper bound for $R_{*}$ is obtained if $T_{\mathrm{I}}$ has a decreasing failure rate.

Calculation of the $R_{*}$ bounds as $T_{\mathrm{E}} \rightarrow \infty$ is achieved as follows. Note that if $T_{\mathrm{E}} \rightarrow \infty$ then $T_{0} \rightarrow T_{\mathrm{I}, 0}$, where $T_{\mathrm{I}, 0}$ denotes the infectious period of the initial infective. Thus, $R_{*}=\lambda_{\mathrm{G}} \mathrm{E}\left[T_{\mathrm{I}}\right]$ if $p_{1}=1$, whilst if $p_{i}=0, i=1,2, \ldots, n$, then, recalling that $D \mid T_{0} \sim \operatorname{Bin}\left(n-1,1-\mathrm{e}^{-\lambda_{\mathrm{L}} T_{0}}\right)$ and using (11), we have $R_{*}=\lambda_{\mathrm{G}} \mathrm{E}\left[T_{\mathrm{I}}\right]\left(1+(n-1)\left(1-\phi_{\mathrm{I}}\left(\lambda_{\mathrm{L}}\right)\right)\right)$, where $\phi_{\mathrm{I}}\left(\lambda_{\mathrm{L}}\right)=\mathrm{E}\left[\mathrm{e}^{-\lambda_{\mathrm{L}} T_{\mathrm{I}}}\right]$.

Finally, consider two epidemics, $E^{(1)}$ and $E^{(2)}$, with a common infectious period distribution but in which the exposure periods in $E^{(1)}$ are stochastically less than those in $E^{(2)}$. It is tempting to conjecture that if $T_{\mathrm{I}}$ has an increasing failure rate, then the severity at the time of the first removal in $E^{(1)}$ exceeds that in $E^{(2)}$, from which results analogous to Corollary 1 would follow. When the household size is $n=2$, the conjecture can be established analytically by arguing as in Lemma 2. For household sizes larger than 2, this approach no longer holds, although numerical calculations continue to support the conjecture.

\section{Concluding comments}

We have provided a general framework for evaluating the usefulness of dynamic vaccination and isolation strategies for SEIR epidemics among a community of households, explored in detail the case of exponentially distributed infectious periods (when the threshold behaviour does not depend on the exposed period distribution) and analysed the effect of the exposed period distribution when the infectious period is not exponentially distributed. The model can be generalised in several ways to make it more realistic. As previously indicated, it is straightforward to extend the analyses of the paper to incorporate unequal household sizes; 
cf. Ball et al. (1997, Section 3.5). The model can be extended to include a random vaccine response, for example by using the framework described by Becker and Starczak (1998), and the exposed period can be split into a vaccine-sensitive period followed by a vaccineinsensitive period, which is appropriate for smallpox. These latter two extensions present no major conceptual difficulties, although calculation of the threshold parameter $R_{*}$ becomes much more involved, even for exponentially distributed infectious periods.

The model can be viewed as incorporating local contact tracing, in that housemates of a removed individual are immediately vaccinated and possibly isolated. This can be extended to global contacts, for example by assuming that a globally contacted individual is traced (and vaccinated) with a given probability when their infector is removed; see Becker et al. (2005), who considered a similar policy for controlling an emerging infectious disease. The analysis of models incorporating such global contact tracing is more complicated and will be considered in a separate paper.

\section{Acknowledgements}

James Pike was supported by an EPSRC studentship. Final revisions to the paper were made whilst Frank Ball was supported by a Leverhulme Research Fellowship.

\section{References}

Andersson, H. And Britton, T. (2000). Stochastic Epidemic Models and Their Statistical Analysis (Lecture Notes Statist. 151). Springer, New York.

Ball, F. G. (1996). Threshold behaviour in stochastic epidemics among households. In Athens Conf. Appl. Prob. Time Ser., Vol. I (Lecture Notes Statist. 114), eds C. C. Heyde et al., Springer, Berlin, pp. 253-266.

BALL, F. G. (1999). Stochastic and deterministic models for SIS epidemics among a population partitioned into households. Math. Biosci. 156, 41-67.

Ball, F. G., Mollison, D. and Scalia-Tomba, G. (1997). Epidemics with two levels of mixing. Ann. Appl. Prob. 7, 46-89.

Ball, F. G., O’Neill, P. D. ANd Pike, J. (2005). Stochastic epidemic models in structured populations featuring dynamic vaccination and isolation. Tech. Rep. 05-04, School of Mathematical Sciences, University of Nottingham.

Becker, N. G. AND StarczaK, D. N. (1998). The effect of random vaccine response on the vaccination coverage required to prevent epidemics. Math. Biosci. 154, 117-135.

Becker, N. G., Glass, K., LI, Z. AND Aldis, G. K. (2005). Controlling emerging infectious diseases like SARS. Math. Biosci. 193, 205-221.

Ethier, S. N. And Kurtz, T. G. (1986). Markov Processes: Characterization and Convergence. John Wiley, New York.

EubANK, S. et al. (2004). Modelling disease outbreaks in realistic urban social networks. Nature 492, 180-184.

Ferguson, N. M. et al. (2005). Strategies for containing an emerging influenza pandemic in Southeast Asia. Nature 437, 209-214.

Halloran, M., Longini, I. M., JR., NiZAm, A. And YAng, Y. (2002). Containing bioterrorist smallpox. Science 298, 1428-1432.

KaPlan, E., Craft, D. AND Wein, L. (2002). Emergency response to a smallpox attack: the case for mass vaccination. Proc. Nat. Acad. Sci. USA 99, 10935-10940.

Longini, I. M., Halloran, M. E., Nizam, A. And Yang, Y. (2004). Containing pandemic influenza with antiviral agents. Amer. J. Epidemiol. 159, 623-633.

PiCARD, P. AND LEFÈvRe, C. (1990). A unified analysis of the final size and severity distribution in collective Reed-Frost epidemic processes. Adv. Appl. Prob. 22, 269-294.

SellKe, T. (1983). On the asymptotic distribution of the size of a stochastic epidemic. J. Appl. Prob. 20, $390-394$.

Watson, R. K. (1980). A useful random time-scale transformation for the standard epidemic model. J. Appl. Prob. 17, 324-332. 